\title{
Complete Pick positivity and unitary invariance
}

\author{
by \\ ANGSHUMAN BHATTACHARYA and \\ TiRTHANKAR BHATTACHARYYA (Bangalore)
}

\begin{abstract}
The characteristic function for a contraction is a classical complete unitary invariant devised by Sz.-Nagy and Foiaş. Just as a contraction is related to the Szegö kernel $k_{S}(z, w)=(1-z \bar{w})^{-1}$ for $|z|,|w|<1$, by means of $\left(1 / k_{S}\right)\left(T, T^{*}\right) \geq 0$, we consider an arbitrary open connected domain $\Omega$ in $\mathbb{C}^{n}$, a complete Pick kernel $k$ on $\Omega$ and a tuple $T=\left(T_{1}, \ldots, T_{n}\right)$ of commuting bounded operators on a complex separable Hilbert space $\mathcal{H}$ such that $(1 / k)\left(T, T^{*}\right) \geq 0$. For a complete Pick kernel the $1 / k$ functional calculus makes sense in a beautiful way. It turns out that the model theory works very well and a characteristic function can be associated with $T$. Moreover, the characteristic function is then a complete unitary invariant for a suitable class of tuples $T$.
\end{abstract}

1. Introduction. Dilation of a contraction to an isometry and further to a unitary operator is a classical result of Sz.-Nagy [19]. Attempts to generalize the results to higher dimensions beyond a pair quickly run into difficulty (see Varopoulos [20]), and hence dilation of a tuple of bounded operators has needed new approaches. While the theory is highly successful for the Euclidean unit ball, little has been done for other domains.

For a contractive tuple or a row contraction, commuting or not, dilation theory has been worked out in great detail in the last two decades (see Arveson [6, Popescu [14] and [15], Müller and Vasilescu [13], and [8] and [9]). For other domains, Ambrozie, Engliš and Müller in [4] and Arazy and Engliš in [5] construct dilations under several conditions on the kernel function. The aim of this note is to show that the theory goes through smoothly for certain complete Pick kernels.

1.1. Kernels and multipliers. Consider a domain $\Omega$ in $\mathbb{C}^{m}$. Let $k(z, w)$ be a positive definite kernel in $\Omega$ which is a holomorphic function in $z$ and an anti-holomorphic function in $w$, and let $\mathbb{H}_{k}$ be the corresponding Hilbert space of holomorphic functions on $\Omega$. A kernel $k$ is called irreducible if

2010 Mathematics Subject Classification: Primary 47A13, 47A48; Secondary 32A70.

Key words and phrases: complete Pick kernels, characteristic function, unitary invariance. 
(1) the functions $k_{w}$, defined by $k_{w}(z)=k(z, w)$ for $w \in \Omega$ are independent, and

(2) $k(x, y)$ is never zero for $x, y \in \Omega$ with $x \neq y$.

All the reproducing kernels will be assumed to be irreducible.

If $\mathcal{E}$ is a Banach space, denote by $\mathcal{O}(\Omega, \mathcal{E})$ the class of all $\mathcal{E}$-valued holomorphic functions on $\Omega$ and by $\mathbb{H}_{k}(\mathcal{E})$ the Hilbert space of $\mathcal{E}$-valued holomorphic functions on $\Omega$ that has the operator valued reproducing kernel $k(z, w) I_{\mathcal{E}}$. For two Hilbert spaces $\mathcal{E}$ and $\mathcal{E}_{*}$, the multiplier space $\mathcal{M}_{k}\left(\mathcal{E}, \mathcal{E}_{*}\right)$ consists of those $\varphi \in \mathcal{O}\left(\Omega, \mathcal{B}\left(\mathcal{E}, \mathcal{E}_{*}\right)\right)$ such that

$$
M_{\varphi}: \mathbb{H}_{k}(\mathcal{E}) \rightarrow \mathbb{H}_{k}\left(\mathcal{E}_{*}\right), \quad f \mapsto \varphi f,
$$

is a continuous linear operator. With the operator norm, the space of multipliers becomes a Banach space, and a Banach algebra if $\mathcal{E}=\mathcal{E}_{*}$. If $\mathcal{E}=\mathcal{E}_{*}$ $=\mathbb{C}$, then we just write $\mathcal{M}_{k}$.

1.2. Pick kernels. The Pick problem for the reproducing kernel Hilbert space $\mathbb{H}_{k}$ is the following. Given $w_{1}, \ldots, w_{n} \in \Omega$ and numbers $\lambda_{1}, \ldots, \lambda_{n}$, is there a $\varphi \in \mathcal{M}_{k}$ of norm at most one which interpolates the data, i.e., satisfies $\varphi\left(w_{i}\right)=\lambda_{i}$ for $i=1, \ldots, n$ ? It is easy to see that a necessary condition for the Pick problem to be solvable is that

$$
\left(1-\lambda_{i} \overline{\lambda_{j}}\right) k\left(w_{i}, w_{j}\right)
$$

is a non-negative definite matrix.

Definition 1.1. The kernel $k$ is called a Pick kernel if the non-negativity of (1.1) is also sufficient to solve the Pick problem.

For the Pick kernels, irreducibility can be assumed without loss of generality by virtue of Lemma 7.2 of [2].

1.3. Complete Pick kernels. Let $\alpha$ and $\beta$ be natural numbers. Given $n$ points $w_{1}, \ldots, w_{n}$ in $\Omega$ and $n$ matrices $\Lambda_{1}, \ldots, \Lambda_{n}$ of size $\alpha \times \beta$, the matrix Pick problem (of order $\alpha \times \beta$ ) is that of finding $\varphi \in \mathcal{M}_{k}\left(\mathbb{C}^{\beta}, \mathbb{C}^{\alpha}\right)$ of norm at most 1 which satisfies $\varphi\left(w_{i}\right)=\Lambda_{i}$ for $i=1, \ldots, n$. Again a necessary condition for solvability of the matrix Pick problem is that the Pick matrix

$$
\left[\left(I-\Lambda_{i} \Lambda_{j}^{*}\right) k\left(w_{i}, w_{j}\right)\right]
$$

is positive (see Theorem 5.8 in [2]).

Definition 1.2. The kernel $k$ is called a complete Pick kernel if positivity of the Pick matrix (1.2) is a sufficient condition for the matrix Pick problem to be solvable for every $\alpha$ and $\beta$.

\subsection{Examples}

(1) Drury-Arveson space. This space, denoted by $H_{d}^{2}$, is defined to be the Hilbert space of holomorphic functions on the Euclidean unit ball in $\mathbb{C}^{d}$ 
with reproducing kernel

$$
k_{d}(z, w)=(1-\langle z, w\rangle)^{-1} .
$$

For more on the space $H_{d}^{2}$ and the multipliers, see the seminal paper of Arveson [6]. The space $H_{d}^{2}$ was first used by Drury [12] who generalized von Neumann's inequality to operator tuples. This kernel is universal among the complete Pick kernels (see [2, Chapter 8]).

(2) Weighted $\ell^{2}$ spaces. Consider a weight sequence $\left(w_{n}\right)_{n \geq 0}$ satisfying

$$
w_{n+1}^{2} \geq w_{n} w_{n+2}
$$

and the space of functions $f(z)=\sum_{n \geq 0} a_{n} z^{n}$ on $\mathbb{D}$ with

$$
\|f\|^{2}:=\sum_{n \geq 0}\left|a_{n}\right|^{2} w_{n}<\infty .
$$

Thus Dirichlet space is a special case, corresponding to $w_{n}=n+1$. These spaces have complete Pick kernels (see [18]).

(3) Dirichlet type spaces. Let $\mu$ be a finite positive measure supported in $\overline{\mathbb{D}}$. Define

$$
D(\mu)=\left\{f: \begin{array}{l}
f \text { is holomorphic in } \mathbb{D} \text { and } \\
\|f\|^{2}:=\|f\|_{H^{2}}^{2}+\int_{\mathbb{D}}\left|f^{\prime}(\zeta)\right|^{2} U_{\mu}(\zeta) d m_{2}(\zeta)<\infty
\end{array}\right\}
$$

where $d m_{2}$ is the normalized Lebesgue area measure in $\mathbb{D}$, and $U_{\mu}$ is defined as

$$
U_{\mu}(\zeta)=\int_{\mathbb{D}} \log \left|\frac{1-\underline{z} \zeta}{\zeta-z}\right|^{2} \frac{d \mu(z)}{1-|z|^{2}}+\int_{\mathbb{T}} \frac{1-|\zeta|^{2}}{|\zeta-z|^{2}} d \mu(z), \quad \zeta \in \mathbb{D} .
$$

These spaces were introduced by Richter [17] and Aleman [3] and were shown to have complete Pick kernel by Shimorin [18].

(4) Weighted Sobolev spaces. Given positive functions $w_{0} \in C\left[x_{0}, x_{1}\right]$ and $w_{1} \in C^{1}\left[x_{0}, x_{1}\right]$, consider the space

$W_{2}^{1}=\left\{f:\left[x_{0}, x_{1}\right] \rightarrow \mathbb{C}: f\right.$ is absolutely continuous with $\left|f^{\prime}\right|^{2}$ integrable $\}$ with the norm

$$
\|f\|^{2}:=\int_{x_{0}}^{x_{1}}|f(x)|^{2} w_{0}(x) d x+\int_{x_{0}}^{x_{1}}\left|f^{\prime}(x)\right|^{2} w_{1}(x) d x .
$$

Then $W_{2}^{1}$ with this norm has a complete Pick kernel. See [16] and [18].

In this note, we shall deal only with complete Pick kernels.

1.5. A complete unitary invariant. In [8], an operator valued holomorphic function on the open Euclidean unit ball $\mathbb{B}_{m}$ in $\mathbb{C}^{m}$ was constructed. This function, called the characteristic function, corresponded to a commuting row contraction, i.e., a tuple of bounded operators $T=\left(T_{1}, \ldots, T_{m}\right)$ on a Hilbert space $\mathcal{H}$ such that $\sum T_{i} T_{i}^{*} \leq I$. The function has the property that 
if two commuting row contractions are unitarily equivalent then their characteristic functions coincide. The converse holds for a class of commuting row contractions. Although in this note we shall consider analogues of what Sz.-Nagy and Foiaş called $C_{.0}$ contractions, in full generality, the converse mentioned above holds for what are called the completely non-coisometric commuting row contractions as was shown in [9].

The aim of this note is to find such a complete unitary invariant as a holomorphic function on $\Omega$ for a much larger class of commuting tuples, viz., those for which $(1 / k)\left(T, T^{*}\right) \geq 0$. These will be called the $(1 / k)$-positive tuples. For how to define $(1 / k)\left(T, T^{*}\right)$, see Section 2 .

2. The space $\mathbb{H}_{k}$ and $(1 / k)$-positive tuples. Let $\Omega \subset \bar{\Omega} \subset \Omega^{\prime} \subset \mathbb{C}^{m}$ be a domain in $\mathbb{C}^{m}$ and $k: \Omega \times \Omega \rightarrow \mathbb{C}$ be a holomorphic complete Pick kernel on $\Omega$. Recall that we assume it is irreducible. Without loss of generality, we also make an assumption that the kernel $k$ is normalized at a point $\lambda_{0}$ in $\Omega$. This means that

$$
k\left(\lambda_{0}, w\right)=k\left(z, \lambda_{0}\right)=1 \quad \text { for all } z, w \in \Omega .
$$

By virtue of general theory of holomorphic complete Pick kernels, there is then a positive integer $d$ (which is not the same as $m$ in general) and a holomorphic function $b=\left(b_{1}, \ldots, b_{d}\right): \Omega \rightarrow \mathbb{B}_{d}$ mapping $\lambda_{0}$ to 0 such that

$$
k(z, w)=\frac{1}{1-\sum_{i=1}^{d} b_{i}(z) \overline{b_{i}(w)}}, \quad z, w \in \Omega .
$$

See Theorem 7.31 in [2]. In general, $d$ need not be finite, i.e., the number of $b_{i}$ could be infinite. However, we make the following assumptions:

(A1) The cardinal $d$ is finite and each $b_{i}$ is holomorphic on $\Omega^{\prime}$ and belongs to the closure of polynomials on $\Omega^{\prime}$ in the compact open topology.

(A2) The coordinate functions $z_{1}, \ldots, z_{m}$ are multipliers on $\mathbb{H}_{k}$.

(A3) The Taylor spectrum $\sigma\left(M_{z}\right)$ is contained in $\Omega^{\prime}$ where $M_{z}=$ $\left(M_{z_{1}}, \ldots, M_{z_{m}}\right)$ is the multiplication tuple on $\mathbb{H}_{k}$.

For a given multi-index $s=\left(s_{1}, \ldots, s_{d}\right)$, let us consider analogues of monomials. Denote by $b^{s}(z)$ the function $b_{1}^{s_{1}}(z) \ldots b_{d}^{s_{d}}(z)$. On the vector space spanned by finite linear combinations of these monomials, we give an inner product by setting

$$
\left\langle b^{s}(z), b^{s^{\prime}}(z)\right\rangle= \begin{cases}0 & \text { if } s \neq s^{\prime}, \\ s ! /|s| ! & \text { if } s=s^{\prime} .\end{cases}
$$

It is then clear that the completion of this linear space with this inner product is $\mathbb{H}_{k}$. Any element $g$ of $\mathbb{H}_{k}$ is of the form $\sum a_{s} b^{s}$ and has the norm $\left(\sum\left|a_{s}\right|^{2} / \gamma_{s}\right)^{1 / 2}$ where $\gamma_{s}$ is the multinomial coefficient for the multi-index $s$. 
Note that this implies that any element $g$ of $\mathbb{H}_{k}$ is of the form

$$
g=f \circ b
$$

for some $f \in H_{d}^{2}$. A straightforward norm computation immediately shows that the $b_{i}$ are contractive multipliers. However, more is true.

Lemma 2.1. $I_{H}-\sum_{i=1}^{d} M_{b_{i}} M_{b_{i}}^{*} \geq 0$.

Proof. Since the linear span of the functions $\left\{k_{w}: w \in \Omega\right\}$ is the whole space, it is enough to show the above just on $k_{w}$. But then

$$
\begin{aligned}
\left(\sum M_{b_{i}} M_{b_{i}}^{*} k_{w}\right)(z) & =\sum b_{i}(z) \overline{b_{i}}(w) \frac{1}{1-\sum b_{i}(z) \overline{b_{i}}(w)} \\
& =\frac{1}{1-\sum b_{i}(z) \overline{b_{i}}(w)}-1=\left(k_{w}-P_{0} k_{w}\right)(z),
\end{aligned}
$$

where $P_{0}$ is the one-dimensional projection onto the constants, thus showing that $I_{H}-\sum_{i=1}^{d} M_{b_{i}} M_{b_{i}}^{*}$ is actually a projection and hence the result.

LEMMA 2.2. $M_{b_{i}}=b_{i}\left(M_{z}\right)$.

Proof. We shall show that $M_{b_{i}}^{*}=\left(b_{i}\left(M_{z}\right)\right)^{*}$ by showing that each member $k_{w}$ of the total set is an eigenvector for $M_{b_{i}}^{*}$ as well as $\left(b_{i}\left(M_{z}\right)\right)^{*}$ with the eigenvalue $\overline{b_{i}(w)}$. Choose, for each $i$, a sequence $\left\{p_{n}^{i}\right\}$ which converges to $b_{i}$ uniformly on compact subsets of $\Omega^{\prime}$ as $n \rightarrow \infty$. The holomorphic functional calculus is a continuous homomorphism (see Theorem 5.18 of Curto [10]). Thus $\left(p_{n}^{i}\left(M_{z}\right)\right)^{*} k_{w} \rightarrow\left(b_{i}\left(M_{z}\right)\right)^{*} k_{w}$ in $\mathbb{H}_{k}$. But $p_{n}^{i}\left(M_{z}\right)=M_{p_{n}}$. So

$$
\begin{aligned}
M_{b_{i}}^{*} k_{w} & =\overline{b_{i}}(w) k_{w}=\lim _{n \rightarrow \infty} \overline{p_{n}^{i}}(w) k_{w}=\lim _{n \rightarrow \infty} M_{p_{n}^{i}}^{*} k_{w} \\
& =\lim _{n \rightarrow \infty}\left(p_{n}^{i}\left(M_{z}\right)\right)^{*} k_{w}=\left(b_{i}\left(M_{z}\right)\right)^{*} k_{w},
\end{aligned}
$$

and that completes the proof.

COROLlaRY 2.3. Let $M_{z}=\left(M_{z_{1}}, \ldots, M_{z_{m}}\right)$ be the multiplication tuple on $\mathbb{H}_{k}$. Then

$$
I_{H}-\sum_{i=1}^{d} b_{i}\left(M_{z}\right)\left(b_{i}\left(M_{z}\right)\right)^{*} \geq 0 .
$$

Proof. This corollary follows from the two lemmas above.

Let $T=\left(T_{1}, \ldots, T_{m}\right)$ denote a commuting $m$-tuple of bounded operators on $\mathcal{H}$ such that $\sigma(T) \subset \Omega$. Define the following tuple via the holomorphic functional calculus of Taylor:

$$
b(T)=\left(b_{1}(T), \ldots, b_{d}(T)\right) .
$$

In this note, we shall be concerned with those tuples $T$ for which $b(T)$ is a $d$-contraction in the sense of Arveson [6]. In other words,

$$
I_{H}-\sum b_{i}(T)\left(b_{i}(T)\right)^{*} \geq 0 .
$$


Since $1 / k$ is a function which is holomorphic in $z$ and anti-holomorphic in $w$ on $\Omega^{\prime}$, there is a well-known method of making sense of $(1 / k)\left(T, T^{*}\right)$ using holomorphic functional calculus (see [11, 4]), which is also related to the hereditary functional calculus of Agler [1]. In our case, $(1 / k)\left(T, T^{*}\right)=$ $I_{H}-\sum b_{i}(T)\left(b_{i}(T)\right)^{*}$ and hence $(1 / k)\left(T, T^{*}\right) \geq 0$. We call such a tuple $T$ a $(1 / k)$-positive tuple. To summarize, our tuple $T$ is assumed to satisfy two conditions:

(C1) $\sigma(T) \subset \Omega$,

(C2) $T$ is $(1 / k)$-positive.

It is clear that the kernel $k$ can be expanded as

$$
k(z, w)=\sum \gamma_{s} b^{s}(z) \overline{b^{s}(w)}
$$

and hence if we define

$$
F_{s}(z, w)=\gamma_{s} \frac{b^{s}(z) \overline{b^{s}(w)}}{k(z, w)} \quad \text { for } z, w \in \Omega
$$

then

$$
\sum_{s \in \mathbb{N}^{d}} F_{s}(z, w)=1
$$

where the sum converges uniformly over compact subsets of $\Omega \times \Omega$. Note that

$$
\begin{aligned}
F_{s}\left(T, T^{*}\right) & =\gamma_{s} b^{s}(T) \frac{1}{k}\left(T, T^{*}\right)\left(b^{s}(T)\right)^{*} \\
& =\gamma_{s} b^{s}(T)\left(I-\sum_{i=1}^{d} b_{i}(T)\left(b_{i}(T)\right)^{*}\right)\left(b^{s}(T)\right)^{*} .
\end{aligned}
$$

By virtue of 2.2 and functional calculus, we have

$$
\sum_{s \in \mathbb{N}^{d}} F_{s}\left(T, T^{*}\right)=I
$$

Since one of our aims here is to produce the dilation of a $(1 / k)$-positive tuple as a functional model, it is natural to look for the smallest space. If $v \in \mathbb{H}_{k}$ denotes the constant function 1 , then $b^{s}\left(M_{z}\right) v$ is orthogonal to $v$ for all multi-indices $s \neq 0$. The following lemma shows that the space $\mathbb{H}_{k}$ is the smallest in the sense that it is contractively contained in other suitable spaces.

Lemma 2.4. Let $T$ be a $(1 / k)$-positive tuple on the Hilbert space $\mathcal{H}$. Let $v \in \mathcal{H}$ be such that $\|v\|=1$ and $b^{s}(T) v \perp v$ for all non-zero $s \in \mathbb{N}^{d}$. Then there exists a contraction $R: \mathbb{H}_{k} \rightarrow \mathcal{H}$ such that $R b^{s}(z)=b^{s}(T) v$. 
Proof. For $s \in \mathbb{N}^{d}$, we know that $\left\|b^{s}(z)\right\|^{2}=s ! /|s|$ !. Consider a polynomial $p(z)=\sum_{|s| \leq r} a_{s} z^{s}$ for some $r \in \mathbb{N}$. We need to prove that

is a contraction. In other words

$$
p(b(z)) \mapsto p(b(T)) v
$$

Now,

$$
\left\|\sum_{|s| \leq r} a_{s} b^{s}(z)\right\|^{2} \geq\left\|\sum_{|s| \leq r} a_{s} b^{s}(T) v\right\|^{2} .
$$

$$
\left\|\sum_{|s| \leq r} a_{s} b^{s}(z)\right\|^{2}=\sum_{|s| \leq r}\left|a_{s}\right|^{2}\left\|b^{s}(z)\right\|^{2},
$$

so, replacing $a_{s}$ by $a_{s} /\left\|b^{s}(z)\right\|^{2}$, we need to prove that

$$
\left\|\sum_{|s| \leq r} \frac{a_{s}}{\left\|b^{s}(z)\right\|} b^{s}(T) v\right\|^{2} \leq \sum_{|s| \leq r}\left|a_{s}\right|^{2} .
$$

Let $E_{0}$ denote the projection onto the one-dimensional subspace spanned by the vector $v \in \mathcal{H}$. Thus, our contractivity condition boils down to proving that

$$
\left\{\frac{a_{s}}{\left\|b^{s}(z)\right\|} b^{s}(T) E_{0}\right\}_{s}
$$

is a contractive tuple. By virtue of the fact that $T$ is a $(1 / k)$-positive tuple, we see that the completely positive map

$$
C_{b(T)}(X)=\sum_{i=1}^{d} b_{i}(T) X\left(b_{i}(T)\right)^{*}
$$

is contractive, i.e., $\left\|C_{b(T)}(I)\right\| \leq 1$. Thus

$$
C_{b(T)}\left(E_{0}\right) \leq C_{b(T)}(I) \leq I .
$$

However, by assumption, $\left\langle b_{i}(T) E_{0} b_{i}(T)^{*} v, v\right\rangle_{H}=0$ so that $C_{b(T)}\left(E_{0}\right) v=0$. This implies that

$$
C_{b(T)}\left(E_{0}\right)+E_{0} \leq I .
$$

Repeated application of $C_{b(T)}(\cdot)$ to the above yields

Now,

$$
E_{0}+C_{b(T)}\left(E_{0}\right)+\cdots+C_{b(T)}^{n}\left(E_{0}\right) \leq I .
$$

$$
C_{b(T)}^{n}=\sum_{|s|=n} \frac{1}{\left\|b^{s}(z)\right\|^{2}} b^{s}(T) E_{0} b^{s}(T)^{*}
$$

because $\left\|b^{s}(z)\right\|^{-2}$ is the multinomial coefficient for the multi-index $s=$ $\left(s_{1}, \ldots, s_{d}\right)$ with $|s|=n$. Therefore we have

$$
\sum_{|s| \leq n} \frac{\left|a_{s}\right|^{2}}{\left\|b^{s}(z)\right\|^{2}} b^{s}(T) E_{0}^{2} b^{s}(T)^{*} \leq I,
$$

and our claim is proved. 
3. The characteristic function. From now, we shall work with only those tuples which satisfy the assumptions $(\mathrm{C} 1)$ and $(\mathrm{C} 2)$.

Recall that a tuple of operators $R=\left(R_{1}, \ldots, R_{d}\right)$ acting on a Hilbert space $\mathcal{L}$ is a $d$-contraction if $\sum R_{i} R_{i}^{*} \leq I_{\mathcal{L}}$. In this case, there are some standard notations:

$$
D_{R^{*}}=\left(I_{\mathcal{L}}-\sum R_{i} R_{i}^{*}\right)^{1 / 2}, \quad \mathcal{D}_{R^{*}}=\overline{\operatorname{Ran}} D_{R^{*}}
$$

and

$D_{R}=\left[\delta_{i j} I_{\mathcal{L}}-R_{i}^{*} R_{j}\right]^{1 / 2} \quad($ a $d \times d$ operator matrix $) \quad$ and $\quad \mathcal{D}_{R}=\overline{\operatorname{Ran}} D_{R}$.

The $d$-contraction $b(T)$, canonically associated with a commuting $(1 / k)$ positive tuple $T$, has its own characteristic function defined by

$$
\theta_{b(T)}(\lambda)=-b(T)+D_{b(T)^{*}}\left(I_{\mathcal{H}}-\lambda b(T)^{*}\right)^{-1} \lambda D_{b(T)}
$$

as a $\mathcal{B}\left(\mathcal{D}_{b(T)}, \mathcal{D}_{b(T)^{*}}\right)$ valued holomorphic function defined on $\mathbb{B}_{d}$. We define the characteristic function of the tuple $T$ to be the following $\mathcal{B}\left(\mathcal{D}_{b(T)}, \mathcal{D}_{b(T)^{*}}\right)$ valued holomorphic function on $\Omega$ :

$$
\theta_{T}(z)=-b(T)+D_{b(T)^{*}}\left(I_{\mathcal{H}}-b(z) b(T)^{*}\right)^{-1} b(z) D_{b(T)} .
$$

Note that $\theta_{T}=\left.\theta_{b(T)}\right|_{b(\Omega)} \circ b$.

LEMMA 3.1. The characteristic function $\theta_{T}$ is a contractive multiplier from $\mathbb{H}_{k} \otimes \mathcal{D}_{b(T)}$ into $\mathbb{H}_{k} \otimes \mathcal{D}_{b(T)^{*}}$. Also, for $z, w \in \Omega$, one has the relation

$$
\begin{aligned}
I-\theta_{T}(z) \theta(w)^{*}= & \left(I-\sum b_{i}(z) \overline{b_{i}(w)}\right) D_{b(T)^{*}} \\
& \times\left(I-\sum b_{i}(z) b_{i}(T)^{*}\right)^{-1}\left(I-\sum b_{i}(T) \overline{b_{i}(w)}\right)^{-1} D_{b(T)^{*}} .
\end{aligned}
$$

Proof. We know that the characteristic function $\theta_{b(T)}$ of the $d$-contraction $b(T)$ is a contractive multiplier from $H_{d}^{2} \otimes \mathcal{D}_{b(T)}$ into $H_{d}^{2} \otimes \mathcal{D}_{b(T)^{*}}$. Also, we have $\theta_{T}=\theta_{b(T)} \circ b$. Thus the first assertion follows from [7, Theorem 3.1].

The second assertion is a straightforward direct computation.

There is a natural unitary map

$$
U: \mathbb{H}_{k} \rightarrow H_{d}^{2}
$$

which sends $b^{s}(z)$ to $\lambda^{s}$ for every multi-index $s$. This $U$ also intertwines $M_{b_{i}}$ on $\mathbb{H}_{k}$ with $M_{z_{i}}$ on $H_{d}^{2}$. So the module $\mathbb{H}_{k}$ formed by the operator tuple $\left(M_{b_{1}}, \ldots, M_{b_{d}}\right)$ is isomorphic to the module $H_{d}^{2}$ formed by the tuple $\left(M_{z_{1}}, \ldots, M_{z_{d}}\right)$ of multiplication by the coordinate functions. Our aim in this note is to study, on $\mathbb{H}_{k}$, the $m$-tuple $\left(M_{z_{1}}, \ldots, M_{z_{m}}\right)$ of multiplication by the coordinate functions.

THEOREM 3.2. The multiplier $M_{\theta_{T}}$ is a partial isometry. 
Proof. We shall show that $M_{\theta_{T}} M_{\theta_{T}}^{*}$ is a projection. To begin, define a linear operator $j: \mathcal{H} \rightarrow \mathbb{H}_{k} \otimes \mathcal{D}_{b(T)^{*}}$ by

$$
j(h)=\sum_{s \in \mathbb{N}^{d}} \gamma_{s} b^{s}(z) \otimes D_{b(T)^{*}} b^{s}(T)^{*} h .
$$

Using the unitary equivalence as above, it can be seen that this $j$ is the same as the corresponding operator in the case of the Drury-Arveson space and it easily follows that

$$
L=j^{*}: \mathbb{H}_{k} \otimes \mathcal{D}_{b(T)^{*}} \rightarrow \mathcal{H}
$$

is the unique bounded operator satisfying

$$
L(p \circ b \otimes \xi)=p(b(T)) D_{b(T) *} \xi
$$

for any polynomial $p$ in $d$ variables.

Any $f$ in $\mathbb{H}_{k}$ has an orthonormal expansion $f=\sum a_{s} b^{s}$. If

$$
p_{n}=\sum_{|s| \leq n} a_{s} b^{s}
$$

then $p_{n}$ converges to $f$ in the topology of $\mathbb{H}_{k}$. On any compact subset of $\Omega$, the modulus of the kernel function $k(z, z)$ is bounded above and bounded away from zero. It follows that $p_{n} \rightarrow f$ uniformly on compact subsets of $\Omega$. Indeed, using the fact that

$$
\left|p_{n}(z)-f(z)\right| \leq \frac{\left\|f-p_{n}\right\|_{\mathbb{H}_{k}}}{1-\left|b_{1}(z)\right|^{2}-\cdots-\left|b_{d}(z)\right|^{2}}
$$

for all $z$ in a compact set, and the fact that the image of a compact set under $b$ is a compact subset of $\mathbb{B}_{d}$, we have uniform convergence of $p_{n}$ to $f$. Since the Taylor spectrum of $T$ is contained in $\Omega$, functional calculus implies that $\left\|p_{n}(T)-f(T)\right\| \rightarrow 0$. Thus we have

$$
\begin{aligned}
L(f \otimes h) & =L\left(\lim _{n} p_{n} \otimes h\right)=\lim _{n} L\left(p_{n} \otimes h\right)=\lim p_{n}(T) D_{b(T)^{*}} h \\
& =f(T) D_{b(T)^{*}} h .
\end{aligned}
$$

This operator is clearly unique. The equation (3.1) immediately gives us that the operator $L$ intertwines $M_{z_{i}} \otimes I$ with $T_{i}$ for every $i$.

There are two important properties of this operator that will be useful. First note that

$$
L(k(\cdot, w) \otimes \eta)=\left(I-\sum_{i} b_{i}(T) \overline{b_{i}(w)}\right)^{-1} D_{b(T)^{*}} \eta
$$

$$
\text { for all } w \in \Omega \text { and } \eta \in \mathcal{D}_{b(T)^{*}} .
$$

Indeed, this is obvious from (3.1) by choosing $f$ to be $k(\cdot, w)$.

The second property is that $L^{*}$ is an isometry. This is a consequence of the Taylor spectrum being contained in $\Omega$. In detail, let $\left\{\eta_{j}\right\}$ be an or- 
thonormal basis of $\mathcal{D}_{b(T)^{*}}$. Then

$$
\begin{aligned}
\left\|L^{*} h\right\|^{2} & =\sum_{s, j}\left|\left\langle L^{*} h, \sqrt{\gamma_{s}} b^{s} \otimes \eta_{j}\right\rangle\right|^{2}=\sum_{s, j}\left|\left\langle h, \sqrt{\gamma_{s}} L\left(b^{s} \otimes \eta_{j}\right)\right\rangle\right|^{2} \\
& =\sum_{s, j}\left|\left\langle h, \sqrt{\gamma_{s}} b^{s}(T) D_{b(T)^{*}} \eta_{j}\right\rangle\right|^{2}=\sum_{s, j}\left|\left\langle\sqrt{\gamma_{s}} D_{b(T)^{*}} b^{s}(T)^{*} h, \eta_{j}\right\rangle\right|^{2} \\
& =\sum_{s}\left\|\sqrt{\gamma_{s}} D_{b(T)^{*}} b^{s}(T)^{*} h\right\|^{2}=\sum_{s}\left\langle\gamma_{s} b^{s}(T) D_{b(T)^{*}}^{2} b^{s}(T)^{*} h, h\right\rangle \\
& =\sum_{s}\left\langle\gamma_{s} b^{s}(T)\left(I-\sum_{i} b_{i}(T) b_{i}(T)^{*}\right) b^{s}(T)^{*} h, h\right\rangle \\
& =\sum_{s}\left\langle F_{s}\left(T, T^{*}\right) h, h\right\rangle=\|h\|^{2} \quad \text { by }(2.3) .
\end{aligned}
$$

Since $L^{*}$ is an isometry, $L^{*} L$ is a projection. The final step in the proof is to show that $L^{*} L$ and $M_{\theta_{T}} M_{\theta_{T}}^{*}$ are orthogonal projections. For $z, w \in \Omega$ and $\xi, \eta \in \mathcal{D}_{b(T)^{*}}$, we have

$$
\begin{aligned}
\left\langle\left( L^{*} L+\right.\right. & \left.\left.M_{\theta_{T}} M_{\theta_{T}}^{*}\right) k(\cdot, z) \otimes \xi, k(\cdot, w) \otimes \eta\right\rangle \\
= & \langle L(k(\cdot, z) \otimes \xi), L(k(\cdot, w) \otimes \eta)\rangle+\left\langle M_{\theta_{T}}^{*}(k(\cdot, z) \otimes \xi), M_{\theta_{T}}^{*}(k(\cdot, w) \otimes \eta)\right\rangle \\
= & \left\langle\left(I-\sum_{i} b_{i}(T) \overline{b_{i}(z)}\right)^{-1} D_{b(T)^{*}} \xi,\left(I-\sum_{i} b_{i}(T) \overline{b_{i}(w)}\right)^{-1} D_{b(T)^{*}} \eta\right\rangle \\
& +\left\langle k(\cdot, z) \otimes \theta_{T}(z)^{*} \xi, k(\cdot, w) \otimes \theta_{T}(w)^{*} \eta\right\rangle \\
= & k(w, z)\left\langle\left(I-\theta_{T}(w) \theta_{T}(z)^{*}\right) \xi, \eta\right\rangle+k(w, z)\left\langle\theta_{T}(w) \theta_{T}(z)^{*} \xi, \eta\right\rangle \\
= & k(w, z)\langle\xi, \eta\rangle=\langle k(\cdot, z) \otimes \xi, k(\cdot, w) \otimes \eta\rangle .
\end{aligned}
$$

This, by virtue of the fact that $\left\{k(\cdot, w) \otimes \eta: w \in \Omega, \eta \in \mathcal{D}_{b(T)^{*}}\right\}$ is a total set in $\mathbb{H}_{k} \otimes \mathcal{D}_{b(T)^{*}}$, shows that $L^{*} L+M_{\theta_{T}} M_{\theta_{T}}^{*}=I_{\mathbb{H}_{k} \otimes \mathcal{D}_{b(T)^{*}}}$, which of course proves the theorem.

4. Unitary invariance. A functional model for a tuple of commuting bounded operators means a Hilbert space $\mathbb{H}$ of holomorphic (possibly vector valued) functions and a subspace $\mathcal{M}$ which is invariant under the multiplication operators by the coordinate functions such that the compressions of the multiplication operators to the orthocomplement of this subspace are simultaneously unitarily equivalent to the given tuple of operators. So let $T=\left(T_{1}, \ldots, T_{m}\right)$ be a tuple of commuting bounded operators on a Hilbert space $\mathcal{H}$. Establishing a functional model for $T$ will mean finding an $\mathbb{H}$ and an $\mathcal{M}$ as above and a Hilbert space isomorphism $U$ between $\mathcal{H}$ and $\mathcal{M}^{\perp}$ such that

$$
U T_{i} U^{*}=\left.P_{\mathcal{M}^{\perp}} M_{z_{i}}\right|_{\mathcal{M}^{\perp}} \quad \text { for all } i=1, \ldots, m .
$$


This can be achieved in the classical case of a contraction $T$ which has the additional property that $\left(T^{*}\right)^{n}$ strongly goes to zero. This means $m=1$, $\Omega=$ the open unit disk and $\mathbb{H}=\mathbb{H}_{k}$ where $k$ is the kernel $k(z, w)=$ $(1-z \bar{w})^{-1}$. More generally, there are functional models for completely nonunitary contractions. In that case, the functional model is more complicated than what is described above, but the essence remains the same. For a detailed account of this, see [19. In several variables, functional models can be successfully constructed for completely non-coisometric commuting contractive tuples, i.e., $m \geq 1$ is an integer, $\Omega$ is the open Euclidean unit ball and $k$ is the Drury-Arveson kernel $k(z, w)=(1-\langle z, w\rangle)^{-1}$. The operator tuple under consideration satisfies

$$
\sum T_{i} T_{i}^{*} \leq I
$$

and is called a contractive tuple for obvious reasons. See [8] and [9]. In the non-commuting situation, this has been done by Popescu for a contractive tuple in [15] where the word function is interpreted in a broader sense.

TheOrem 4.1. Consider the space

$$
\mathbb{H}_{T}=\left(\mathbb{H}_{k} \otimes \mathcal{D}_{b(T)^{*}}\right) \ominus \operatorname{Ran} M_{\theta_{T}}
$$

and the operator tuple on $\mathbb{H}_{T}$ defined for $i=1, \ldots, m$ by

$$
\tilde{T}_{i}=\left.P_{\mathbb{H}_{T}}\left(M_{z_{i}} \otimes I\right)\right|_{\mathbb{H}_{T}}
$$

The given tuple $T$ is unitarily equivalent to $\tilde{T}$.

Proof. Since $L^{*}$ is an isometry, it is clear that $T_{i}$ is unitarily equivalent to $L^{*} T_{i} L$ acting on $L^{*} \mathcal{H}$. The fact that $L^{*} L$ and $M_{\theta_{T}} M_{\theta_{T}}^{*}$ are orthogonal projections then finishes the proof.

The notion of coincidence of operator valued holomorphic functions goes back to Sz.-Nagy. Given two $(1 / k)$-positive tuples $T$ and $R$ on Hilbert spaces $\mathcal{H}$ and $\mathcal{K}$, the characteristic functions of $T$ and $R$ are said to coincide if there exist unitary operators $\tau: \mathcal{D}_{b(T)} \rightarrow \mathcal{D}_{b(R)}$ and $\tau_{*}: \mathcal{D}_{b(T)^{*}} \rightarrow \mathcal{D}_{b(R)^{*}}$ such that the following diagram commutes for all $z$ in $\Omega$ :

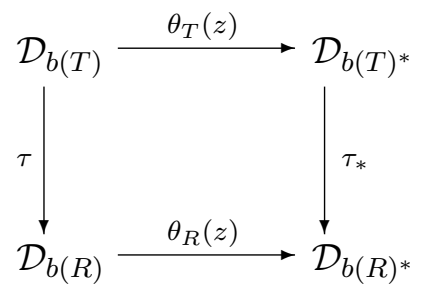

The notion is important because if $T$ and $R$ are unitarily equivalent, i.e., if there is a unitary $U: \mathcal{H} \rightarrow \mathcal{K}$ such that $U T_{i}=R_{i} U$ for all $i=$ $1, \ldots, m$, then $U p(T)=p(R) U$ for all polynomials $p \in \mathbb{C}\left[z_{1}, \ldots, z_{m}\right]$. By 
approximating each $b_{i}$ on compact subsets of $\Omega^{\prime}$ by a sequence of polynomials $\left\{p_{n}^{i}\right\}_{n=1}^{\infty}$, say, we have, by functional calculus,

$$
U b_{i}(T)=U \lim _{n} p_{n}^{i}(T)=\lim _{n} U p_{n}^{i}(T)=\lim _{n} p_{n}^{i}(R) U=b_{i}(R) U .
$$

Thus the $d$-contractions $b(T)$ and $b(R)$ are unitarily equivalent. Thus their characteristic functions coincide (see [8, Proposition 4.2]). As $\theta_{T}=\theta_{b(T)} \circ b$, we see that $\theta_{T}$ and $\theta_{R}$ coincide. It is the converse that is non-trivial and uses the assumption $(\mathrm{C} 1)$ crucially.

THEOREM 4.2. The characteristic functions $\theta_{T}$ and $\theta_{R}$ coincide if and only if the tuples $T$ and $R$ are unitarily equivalent.

Proof. Let $\tau^{\prime}: \mathcal{D}_{b(T)} \rightarrow \mathcal{D}_{b(R)}$ and $\tau_{*}^{\prime}: \mathcal{D}_{b(T)^{*}} \rightarrow \mathcal{D}_{b(R)^{*}}$ be two unitary operators such that

$$
\tau_{*}^{\prime} \theta_{T}(z)=\theta_{R}(z) \tau^{\prime} \quad \text { for all } z \in \Omega .
$$

The operators $\tau^{\prime}$ and $\tau_{*}^{\prime}$ give rise to unitary operators

$$
\tau: \mathbb{H}_{k} \otimes \mathcal{D}_{b(T)} \rightarrow \mathbb{H}_{k} \otimes \mathcal{D}_{b(R)} \quad \text { and } \quad \tau_{*}: \mathbb{H}_{k} \otimes \mathcal{D}_{b(T)^{*}} \rightarrow \mathbb{H}_{k} \otimes \mathcal{D}_{b(R)^{*}}
$$

Checking the action on elementary tensors, we get

$$
M_{\theta_{R}} \tau=\tau_{*} M_{\theta_{T}} .
$$

This is the key relation which shows that $\left.\tau_{*}\right|_{\mathbb{H}_{T}}: \mathbb{H}_{T} \rightarrow \mathbb{H}_{R}$ is a unitary operator which intertwines the model tuples $\tilde{T}$ and $\tilde{R}$. But then, by Theorem 4.1. we conclude that $T$ and $R$ are unitarily equivalent.

5. The Toeplitz $C^{*}$-algebra. The $C^{*}$-algebra generated by the unilateral shift on $H^{2}$ of the disk plays a major role in the theory of dilation of a single contraction. Indeed, given a contraction $T$ on a Hilbert space $\mathcal{H}$, the canonical completely positive map $\varphi: C^{*}(S) \rightarrow \mathcal{B}(\mathcal{H})$ which sends $S$ to $T$ can be thought of as the model. Its dilation to a representation $\pi$ gives the dilation of $T$. This method of proof has the advantage that it is readily applicable to the case of an operator tuple.

The Toeplitz $C^{*}$-algebra $\mathcal{T}_{m}^{k}$ in $m$ variables dependent on the kernel $k$ is the $C^{*}$-subalgebra of $\mathcal{B}\left(\mathbb{H}_{k}\right)$ generated by the multiplication operators $M_{z_{1}}, \ldots, M_{z_{m}}$ acting on $\mathbb{H}_{k}$. By virtue of (2.1), we know that there are $m$ functions $f_{1}, \ldots, f_{m} \in H_{d}^{2}$ defined in the unit ball of $\mathbb{C}^{d}$ such that the coordinate function $z_{i}$ in $\mathbb{C}^{m}$ is given by $z_{i}=f_{i} \circ b$.

Lemma 5.1. The functions $f_{i}$ are multipliers on $H_{d}^{2}$.

Proof. Let $g \in H_{d}^{2}$. Then $g \circ b \in \mathbb{H}_{k}$. Since we have assumed that $z_{i}$ are multipliers on $\mathbb{H}_{k}$, we have $z_{i}(g \circ b) \in \mathbb{H}_{k}$. Now

$$
z_{i}(g \circ b) \in \mathbb{H}_{k}=f_{i} \circ b(g \circ b)=\left(f_{i} g\right)(b(z)) .
$$

It is clear that $\left\|z_{i}(g \circ b)\right\|_{\mathbb{H}_{k}}^{2}=\left\|f_{i} g\right\|_{H_{d}^{2}}^{2}$ and hence the conclusion. 
We end with the following result which is interesting in its own right.

LEMMA 5.2. If $\mathbb{B}_{d}$ is not the domain of holomorphy for $f_{1}, \ldots, f_{m}$, then the $C^{*}$-algebra $\mathcal{T}_{m}^{k}$ is isomorphic to the Toeplitz $C^{*}$-algebra $\mathcal{T}_{d}^{k_{d}}$.

REMARK. The isomorphism actually goes via the unitary $U$ from the beginning of Section 3 .

Proof of Lemma 5.2. We first show that there is an isometric embedding of the Toeplitz $C^{*}$-algebra $\mathcal{T}_{d}^{k_{d}}$ generated by the coordinate multipliers on the Drury-Arveson space $H_{d}^{2}$ inside $\mathcal{T}_{m}^{k}$. This is true in general, i.e., without the hypothesis of the lemma.

By assumption (A1), for each $i$, there is a sequence $\left\{p_{n}^{i}\right\}_{n=1}^{\infty}$ of polynomials in $m$ variables such that $p_{n}^{i} \rightarrow b_{i}$ as $n \rightarrow \infty$ uniformly on compact subsets of $\Omega^{\prime}$. Then applying the continuity of functional calculus and noting that $b_{i}\left(M_{z_{1}}, \ldots, M_{z_{m}}\right)=M_{b_{i}}$, we see that $p_{n}^{i}\left(M_{z}\right) \rightarrow M_{b_{i}}$ as $n \rightarrow \infty$ in the norm topology of $\mathcal{B}\left(\mathbb{H}_{k}\right)$. Thus $M_{b_{1}}, \ldots, M_{b_{m}}$ are members of the $C^{*}$-algebra $\mathcal{T}_{m}^{k}$. So the $C^{*}$-algebra generated by $M_{b_{1}}, \ldots, M_{b_{m}}$ is contained in $\mathcal{T}_{m}^{k}$. Since the $C^{*}$-algebra generated by the $b_{i}$ is isomorphic to $\mathcal{T}_{d}^{k_{d}}$, we deduce that a copy of $\mathcal{T}_{d}^{k_{d}}$ is contained in $\mathcal{T}_{m}^{k}$.

For the reverse inclusion, we need the assumption that $\mathbb{B}_{d}$ is not the domain of holomorphy for any of the $f_{i}$ because then we get a bigger ball where the $f_{i}$ are holomorphic. Thus we have power series expansions for them. For each $i$, the power series for $f_{i}$ converges to $f_{i}$ uniformly on compact subsets of this bigger ball. So we get a polynomial sequence $\left\{q_{n}^{i}\right\}_{i \geq 1}$ such that $q_{n}^{i} \rightarrow f_{i}$ uniformly on compact subsets of this bigger ball. Recall that the Taylor spectrum of the Drury-Arveson shift is contained the closed Euclidean unit ball. So we can apply holomorphic functional calculus (in a way similar to the proof of Lemma 2.2) and find that $M_{q_{n}^{i}} \rightarrow M_{f_{i}}$ in operator norm, showing that $M_{f_{i}}$ is in $\mathcal{T}_{d}^{k_{d}}$. So the $C^{*}$-algebra generated by $M_{f_{1}}, \ldots, M_{f_{m}}$ is contained in $\mathcal{T}_{d}^{k_{d}}$. But the $C^{*}$-algebra generated by $M_{f_{1}}, \ldots, M_{f_{m}}$ is isomorphic to $\mathcal{T}_{m}^{k}$. That completes the proof.

Let $\mathcal{A}_{m}^{k}$ denote the subalgebra of $\mathcal{B}\left(\mathbb{H}_{k}\right)$ generated by $M_{z_{1}}, \ldots, M_{z_{m}}$. Then it is clear from the lemma above that in the case when $\mathbb{B}_{d}$ is not the domain of holomorphy for $f_{1}, \ldots, f_{m}$, we have $\mathcal{T}_{m}^{k}=\overline{\operatorname{span}} \mathcal{A}_{m}^{k}\left(\mathcal{A}_{m}^{k}\right)^{*}$.

Acknowledgements. Work of the second named author is supported by Ramanna Fellowship from DST and UGC SAP Phase IV. The authors are thankful to the referee for a careful reading.

\section{References}

[1] J. Agler, The Arveson extension theorem and coanalytic models, Integral Equations Operator Theory 5 (1982), 608-631. 
[2] J. Agler and J. E. McCarthy, Pick Interpolation and Hilbert Function Spaces, Grad. Stud. Math. 44, Amer. Math. Soc., Providence, RI, 2002.

[3] A. Aleman, The multiplication operator on Hilbert spaces of analytic functions, Habilitationsschrift, Fernuniversität Hagen, 1993.

[4] C.-G. Ambrozie, M. Engliš and V. Müller, Operator tuples and analytic models over general domains in $\mathbb{C}^{n}$, J. Operator Theory 47 (2002), 287-302.

[5] J. Arazy and M. Engliš, Analytic models for commuting operator tuples on bounded symmetric domains, Trans. Amer. Math. Soc. 355 (2003), 837-864.

[6] W. Arveson, Subalgebras of $C^{*}$-algebras III: Multivariable operator theory, Acta Math. 181 (1998), 159-228.

[7] J. A. Ball, T. T. Trent and V. Vinnikov, Interpolation and commutant lifting for multipliers on reproducing kernel Hilbert spaces, in: Operator Theory and Analysis: The M. A. Kaashoek Anniversary Volume (Amsterdam, 1997), Operator Theory Adv. Appl. 122, Birkhäuser, Basel, 2001, 89-138.

[8] T. Bhattacharyya, J. Eschmeier and J. Sarkar, Characteristic function of a pure commuting contractive tuple, Integral Equations Operator Theory 53 (2005), 23-32.

[9] - - - - On CNC commuting contractive tuples, Proc. Indian Acad. Sci. Math. Sci. 116 (2006), 299-316.

[10] R. E. Curto, Applications of several complex variables to multiparameter spectral theory, in: Surveys of Some Recent Results in Operator Theory, Vol. II, Pitman Res. Notes Math. Ser. 192, Longman Sci. Tech., Harlow, 1988, 25-90.

[11] R. E. Curto and L. A. Fialkow, The spectral picture of $\left(L_{A}, R_{B}\right)$, J. Funct. Anal. 71 (1987), 371-392.

[12] S. W. Drury, A generalization of von Neumann's inequality to the complex ball, Proc. Amer. Math. Soc. 68 (1978), 300-304.

[13] V. Müller and F.-H. Vasilescu, Standard models for some commuting multioperators, ibid. 117 (1993), 979-989.

[14] G. Popescu, Isometric dilations for infinite sequences of noncommuting operators, Trans. Amer. Math. Soc. 316 (1989), 523-536.

[15] - Characteristic functions for infinite sequences of noncommuting operators, J. Operator Theory 22 (1989), 51-71.

[16] P. Quiggin, For which reproducing kernel Hilbert spaces is Pick's theorem true?, Integral Equations Operator Theory 16 (1993), 244-266.

[17] S. Richter, A representation theorem for cyclic analytic two-isometries, Trans. Amer. Math. Soc. 328 (1991), 325-349.

[18] S. Shimorin, Complete Nevannlinna-Pick property of Dirichlet-type spaces, J. Funct. Anal. 191 (2002), 276-296.

[19] B. Sz.-Nagy and C. Foias, Harmonic Analysis of Operators on Hilbert Space, NorthHolland, Amsterdam, American Elsevier, New York, and Akadémiai Kiadó, Budapest, 1970.

[20] N. Varopoulos, On an inequality of von Neumann and an application of the metric theory of tensor products to operator theory, J. Funct. Anal. 16 (1974), 83-100.

Angshuman Bhattacharya, Tirthankar Bhattacharyya

Indian Institute of Science

Bangalore 560012, India

E-mail: angshu@math.iisc.ernet.in tirtha@member.ams.org 